
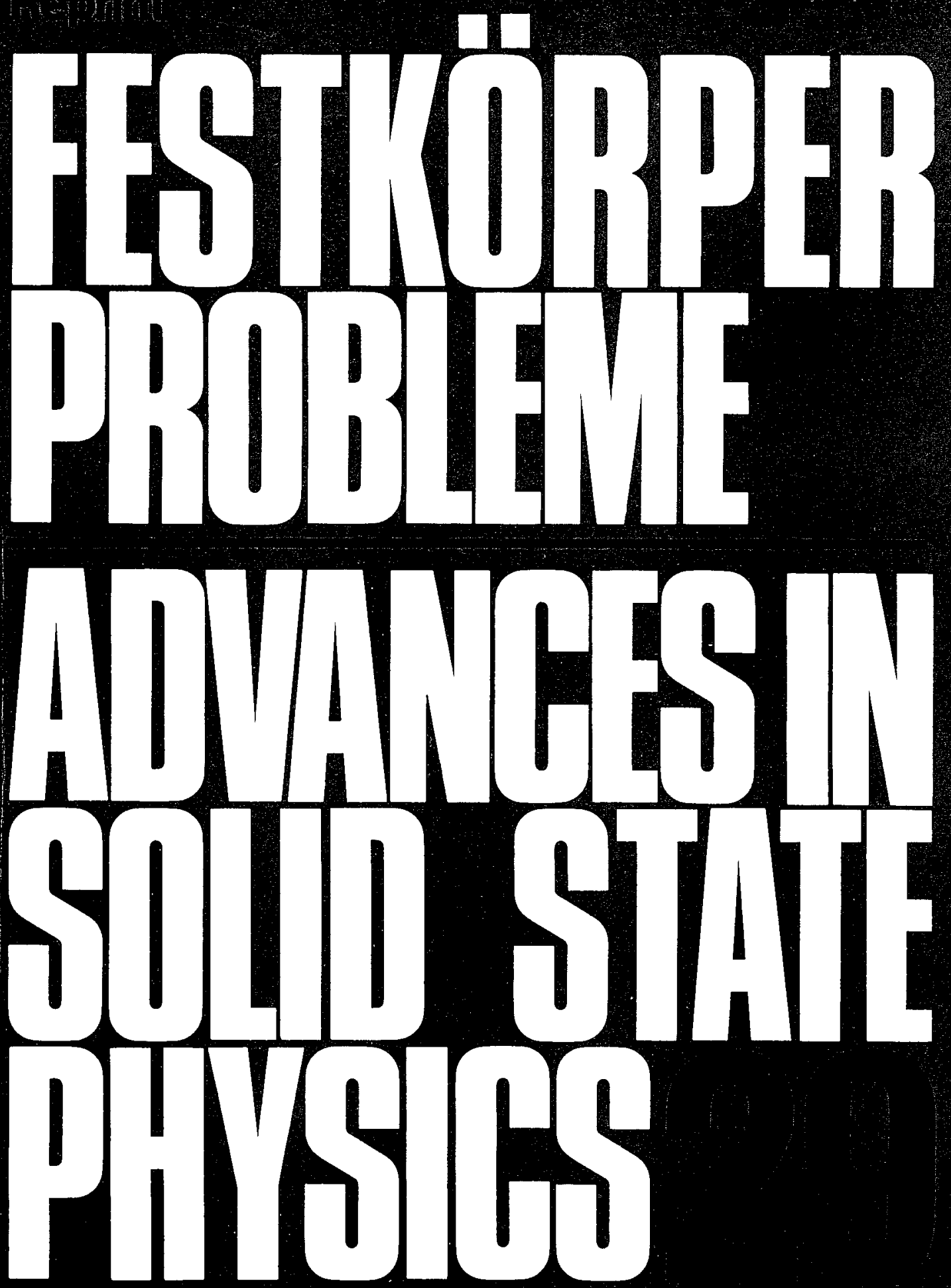


\section{Contents}

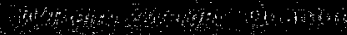

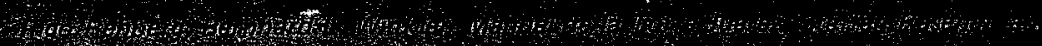

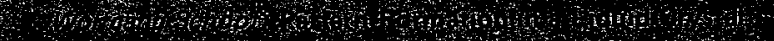

on

m ans

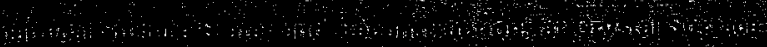

Q 


\title{
Coherent electron focusing
}

\author{
C. W. J. Beenakker
}

Philips Research Laboratories, 5600 JA Eindhoven, The Netherlands

\section{H. van Houten}

Philips Laboratories, Briarcliff Manor, NY 10510, USA

\section{B. J. van Wees}

Applied Physics, Delft University of Technology, 2600 GA Delft, The Netherlands

\begin{abstract}
Summary: Theory and experiment are reviewed of the classical and guantum mechanical focusing by a magnetic ficlel of baltistic clectrons injected through a point contact in a two-dimensional electron gas. Two alternative points of vicw are cmphasized. On the one hand, the experiment is a realization of electron opties in the solid state. The three basic building blocks are a coherent and monochromatic point source/detector, an electrostatic mirror with little diffuse scatlering, and a magnetic lens. On the other hand, coherent electron focusing is a resistance measurement in the guantum ballistic transport regime, which exhibits the characteristic features of this regime in a most extreme way. For cxample, large magnetoresistance oscillations occur (up to 95\% amplitude modulation is observed), with a periodicity which is non-locally determined by the separation between eurrent and voltage point contacts. A WKB calculation of the thansmission probabilitics shows that this effect is the result of the interference of coherently excited magnetic edge states at the clectron gas boundary. Another example is the absence of local equilibrium: The measurements show that the point contacts can selectively populate (and detect) specific Landau levels, and that this highly non-cepulibrium population is maintained over distances of microns.
\end{abstract}

\section{Introduction}

Electron focusing in metals was pioneered by Sharvin [1] and Tsoi [2] as a powerful tool to investigate the shape of the Fermi surface, surface scattering, and the electron-phonon interaction 131. The experiment is the analogue in the solicl state of magnetic focusing in vacuum (e.g. in a $\beta$-spectrometer). Required is a large mean rree path for the carriers at the Fermi surface, to ensure ballistic motion as in vacuum. The mean free path (which can be as large as $1 \mathrm{~cm}$ in pure melallic single crystals) should be much larger than the length $l$ on which the focusing lakes place. Experimentally $L=10^{-2}-10^{-1} \mathrm{~cm}$ is the separation of two metallic needles (point contacts) pressed on the crystal surface, which serve to inject a divergent electron beam and detect its focusing by the mag- 
netic field. In metals, electron focusing is essentially a classical phenomenon because of the small Fermi wave length $\lambda_{1}$ (typically $0.5 \mathrm{~nm}$, on the order of the inter-atomic separation).

The Fermi wave length is 100 times as large in the two-dimensional electron gas (2DEG) which is present at the interface of a GaAs-AlGaAs heterostructure. This length scale is within reach of electron-beam lithography, while remaining well below the mean fiee path in high-mobility materal $(10 \mu \mathrm{m}$ can be tealized at low temperatures in heterostructures grown by molecular-beam epitaxy). For these two reasons the quantum ballistic transpot tegime has become accessible in a $2 D E G$ [4]. In the present paper theory and experiment are reviewed of electron focusing in this regime [5-8], which turns out to be strikingly different from the classical tegime familiar from metals. This has motivated the new name: coherent clectron focusing.

The geometiy of the experiment (Fig. 1) is the transverse focusing geometry of Tsoi [2], and consists of two point contacts on the same boundary in a perpendicular magnetic freld $B$. [ In metals one can also use the geometry of Sharvin [1], with opposite point contacts in a longitudinal field. This is not possible in two dimensions. ] Because the electron gas is confined to the interior of the heterostructurc, one can not just use a metal needle to fabricate a point contact to a $2 \mathrm{DEF}$. Instead, the point contacts are created electrostatically by depositing an electrode of a sutable shape on top of the heterostructure [9]. On applying a negative vollage to the split-gate electrode shown in Fig. I the electron gas underneath the gate structure is depleted, crealing two 2DEG regions ( $i$ and c) electically isolated from the rest of the $2 D E G$ - apart from the two narrow and short constrictions (point contacts)
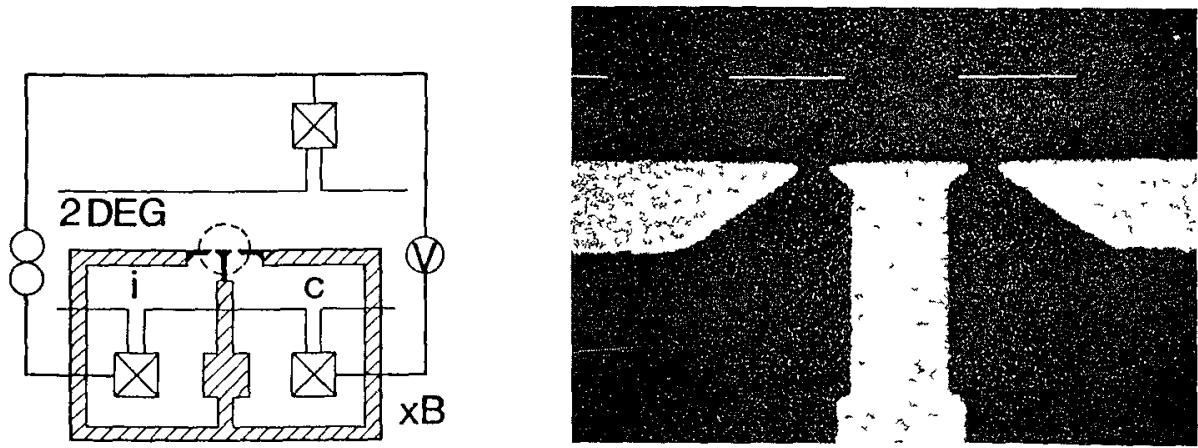

Fig. 1 Schematic layout (left) of the double point entatet device for the electson focusing experiments (in a threc-terminal measurement configusation). The coossed squares are ohmic contacts to the 2 DEG. The split-gate (shaded) sepalates injector (i) and collector (c) ateas fiom the butk 2DEG. The fine details of the gate structure inside the dashed circle arc shown in a scannng clectson microglaph (aght). The bar denotes a length of $1 \mu \mathrm{m}$. In this device the point contact separation is $L=1.5$ $\mu \mathrm{m}$. A device with $L=3.0 \mathrm{\mu m}$ was also studied. [ From Ref 8. ] 
under the $250 \mathrm{~nm}$ wide openings in the split-gate. The devices studied had point contact separations $L$ of 1.5 and $3.0 \mu \mathrm{m}$, both values being below the mean free path of $9 \mathrm{\mu m}$ estimated from the mobility. Because the depletion potential extends laterally beyond the gate pattern for high (negative) gate vollages, one can force the constrictions to become progressively narrower (at the same time reducing the electron gas density in the constrictions) - until they are fully pinched off. By this technique it is possible to create point contacts of variable width $W$, something which is not realizable in a metal. Note that $W$ is comparable in magnitude to $\lambda_{\Gamma}$ (which was $40 \mathrm{~nm}$ in the devices studied). These are quantum point contacts, as evidenced by their conductance which was discovered to be approximately quantized in units of $\left.2 c^{2} / h \mid 10,11\right]$.

Electron focusing can be seen as a transmission experiment in electron optics. The classical regime then corresponds to geometrical optics, the guantum regime to wave optics. The optical analogy is useful, both to understand the experiments and to inspire new ones [12]. An alternative point of view is that coherent electron focusing is a prototype of a non-local resistance measurement in the quantum ballistic transport regime, such as studied extensively in narrow-channel geometries $|13|$. Longitudinal resistances which are negative, not $\pm B$ symmetric, and dependent on the properties of the current and voltage contacts as well as on their separation; periodic and aperiodic magnetoresistance oscillations; absence of local equilibrium - these are all characteristic features of this transport regime which appear in a most extreme and bare form in the electron focusing geometry. One reason for the simplification offered by this geometry is that the current and voltage contacts, being point contacts, are not nearly as invasive as the wide leads in a Ilall bar geometry [14]. Another reason is that the electrons interact with only one boundary (instead of two in a narrow channel).

The outline of this paper is as follows. In Sec. 2 the experimental results on electron rocusing $[5,8]$ are described as a transmission experiment in a 2DEG. $\triangle$ theoretical description $|6,8|$ is given in Sec. 3 , in terms of mode interference in the wave guide formed by the magnetic field at the 2DEG boundary. In Sec. 4 we discuss the quantum IIall efrect in the electron focusing genmetry $[7,8]$ as a non-local resistance measurement. The theoretical framework used to relate these two alternative descriptions is the Landater-Bütliker formalism $|15,16|$, which treats a resistance measurement as a transmission experiment. We conclude in Sec. 5 .

\section{Mirror, lens, and point source}

Fig. 2 illustrates electron focusing in two dimensions as it follows from classical mechanics. The arrangement combines three hasic elements: mirror, magnetic lens, and point source/detector. The point source (i) injects electrons with the Fermi energy $E_{1} \equiv m v_{1}{ }^{2} / 2$ ballistically into the 


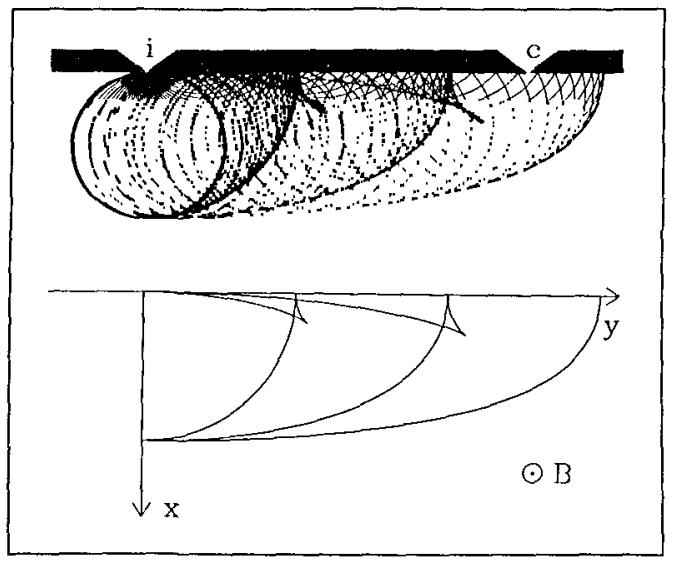

Fig. 2

Top: Skipping orbits along the 2DEG bounclary. The trajectorics are drawn up to the third specular reflection. Bottom: Plot of the calustics, which are the collection of focal points of the trajectorics. [From Ref 8. ]

2DEG. The injected electrons all have the same Fermi velocity $v_{\text {I }}$, hut in different directions. Electrons are detected if they reach the adjacent collector (c), after one or more specular reflections at the boundary connecting $i$ and $c$. These skipping orbits are composed of translated circular arcs of identical radius $l_{\text {cycl }} \equiv h k_{\mathrm{F}} / e B$, which is the cyclotron radius in a perpendicular magnetic field $B\left(k_{\mathrm{F}} \equiv m \mathrm{v}_{\mathrm{l}} / h_{i}\right.$ is the Fermi wave vector). The focusing action of the magnetic field is evident in Fig. 2 (top) from the black lines of high density of trajectories. These lines are known in optics as canstics, and are plotied separately in $\Gamma$ ig. 2 (bottom). The caustics intersect the $2 \mathrm{DEG}$ boundary at multiples of the cyclotron diameter from the injector. As the magnetic field is increased, a series of these focal points shifts past the collector. The electron flux incident on the collector thus reaches a maximum whenever its separation $L$, from the injector is an integer multiple of $2 l_{\text {cycl }}$. This occurs when $B=p B_{\text {focus }}, p=1,2, \ldots$, with

$B_{\text {focus }}=2 h k_{\mathrm{I}} / e L$.

For a given injected current $l_{i}$ the voltage $V_{c}$ on the collector is proportional to the incident flux. The classical picture thus predicts a series of equidistant peaks in the collector voltage as a function of magnetic field.

In Fig. 3 (top) we show such a classical focusing spectrum, calculated for parameters corresponding to the experiment discussed below $\left(L=3.0 \mu \mathrm{m}, k_{\mathrm{r}}=0.15 \mathrm{~nm}^{-1}\right)$. The spectrum consists of equidistant focusing peaks of approximately equal magnitude superimposed on the Ilall resistance (dashed line). The $p$-th peak is due to electrons injected perpendicularly to the boundary which have made $p-1$ specular reflections between injector and collector. Such a classical focusing spectrum is commonly observed in metals [17], albeit with a decreasing 


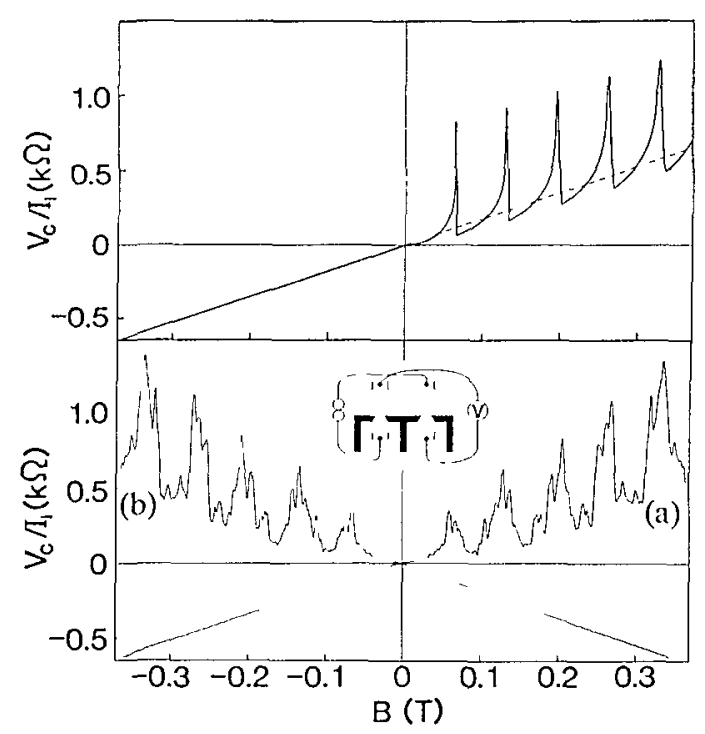

Fig.3

Botlom: Experimental electron focusing spectrum $\left(T^{\prime}=50 \mathrm{mK}\right.$, $L=3.0 \mu \mathrm{m})$ in the generalized Hall resistance configuration depicted in the inset. The two Iraces $a$ and $b$ are measured with interchanged current and voltage leads, and demonstratc the injector-collector reciprocity as well as the reproducibility of the fine structure. Top: Calculated classical focusing spectrum corresponding to the experimental trace a $(50 \mathrm{~nm}$ wide point contacts wete assumed). The dashed line is the extrapolation of the classical llall tesistance seen in reverse ficlds. [ From Ref 8.]

height of subsequent peaks because of partially diffuse scattering at the metal surface. Note that the peaks occur in one field direction only; In reverse fields the focal points are at the wrong side of the injector for detection, and the normal IIall resistance is obtained. The experimental result for a $2 \mathrm{DEG}$ is shown in the bottom half of Fig. 3 (trace $a$; trace $b$ is discussed below). A series of five focusing peaks is evident at the expected positions. This observation by itself has two important implications:

- A point contact acts as a monochromatic point source of ballistic electrons with a well-clefined energy;

- The electrostatically defined 2DEG boundary is a good mirror with little diffuse scattering.

Fig. 3 is obtained in a measuring configuration (insel) in which an imaginary line connecting the voltage probes crosses that between the current source and drain. This is the configuration for a generalized I I all resistance measurement. Alternatively, one can measure a generalized longitudinal resistance, in the configuration shown in the inset of Fig. 4. One then measures the focusing peaks without a superimposed Hall slope. Note that the experimental longitudinal resistance (Fig. 4, bottom) becomes negative. This is a classical result of magnetic focusing, as demonstrated by the calculation shown in the top half of Fig. 4. Büttiker [18] has studied negative longitudinal resistances in a different (Ilall bar) geometry.

On the experimental focusing poaks a finc structure is evident in rigs. 3 and 4 . The rine structure is well reproducible (compare Figs. 3 and 


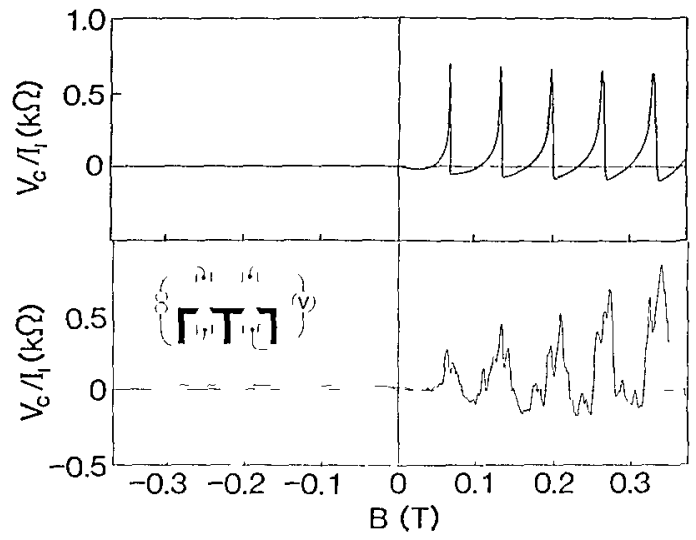

Fig.4

As Fig. 3, but in the longitudinal tcsistince configuration. [ From Ref 8.1

4), but sample dependent. It is only resolved at low temperatures (below $1 \mathrm{~K}$ ) and small injection voltages (the measurements shown are taken at $50 \mathrm{mK}$ and a few $\mu \mathrm{V} \wedge \mathrm{C}$ voltage over the injector). A nice demonstration of the reproducibility of the fine structure is oblained upon interchanging current and voltage leads, so that the injector becomes the collector and vice versa. The resulting focusing spectrum shown in Fig. 3 (trace $b$ ) is almost the precise mirror image of the original one (trace a) - although this particular device had a strong asymmetry in the widths of injector and collector. The symmetry in the focusing spectra is a consequence of the fundamental reciprocity relation derived by Büttiker [16], which generalizes the familiar Onsager-Casimir symmetry relation for the resistivity tensor to resistances (see Sec. 4).

The fine structure on the focusing peaks in Figs. 3 and 4 is the first indication that electron focusing in a $2 D E G$ is qualitatively different from the corresponding experiment in metals. At higher magnetic fields the resemblance to the classical focusing spectrum is lost, sce rig. 5. A Fourier transform of the spectrum for $B \geq 0.8 \mathrm{~T}$ (inset in Fig. 5) shows that the large-amplitude high-field oscillations have a dominant periodicity of $0.1 \mathrm{~T}$, which is approximately the same as the periodicity $B_{\text {tous }}$ of the much smaller focusing peaks at low magnetic fields ( $B_{\text {focus }}$ in Fig. 5 differs from Fig. 3 because of a smaller $L=1.5 \mu \mathrm{m}$ ). This dominant periodicity is the result of quantum interference between the different trajectories in Fig. 2 which take an electron from injector to collector. [In Sec. 3 we show this in a mode picture, which in the WKB approximation is equivalent to calculating the interferences of the (complex) probability amplitude along classical trajectories. The latter ray picture is treated extensively in Ref. 8. J The theoretical analysis implies for the experiment that:

- The injector acts as a coherent point source with the coherence maintained over a distance of several microns to the collector. 


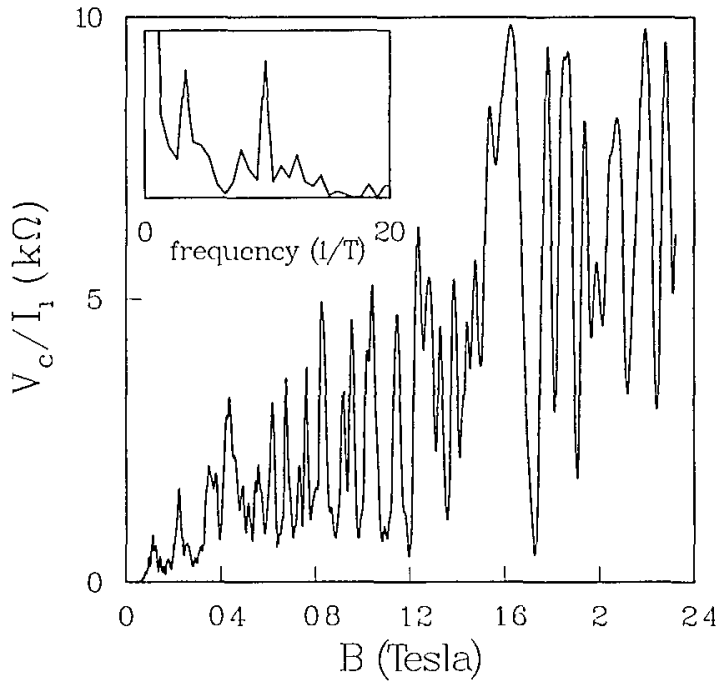

Fig.5

Experimental clectron focusing spectrum over a larger fick range and for very narrow point contacts (estimated willth $20-40 \mathrm{~nm} ; T=50 \mathrm{mK}$, $L=1.5 / \mathrm{mm}$ ). The inset gives the Foutice transform for $B \geq 0.8 \mathrm{~T}$. The high-ficld oscillations bave the same dominant periodicity as the low-ficld focusing peaks - but with a much larger amplitude. [ From Rer 8.]

\section{Edge states and skipping orbits}

Magnetic edge states $[19,20]$ are transverse modes of a wave guide of width $\sim l_{\text {cycl }}$ formed by the magnetic field at the 2DEG boundary. The edge states at the Fermi level are labelled by a quantum number $n=1,2 \ldots N$, with $N=k_{\mathrm{F}} l_{\text {cycl }} / 2$ the total number of propagating modes or edge channels (for simplicity we ignore here the discreteness of $N$ ). An injector of width below $\lambda_{1}$ : excites a coherent superposition of these propagating modes (plus evanescent modes, which using the ray treatment of Ref. 8 are found to give only a small contribution for the large $k_{1} . L$ considered, and will be neglected here). The wave function $\Psi$ is of the form

$\Psi(x, y)=\sum_{n=1}^{N} a_{n} f_{n}(x) \mathrm{e}^{\mathrm{i} k_{n}, y}$.

IIere $k_{n}$ is the wave number for propagation of mode $n$ in the $y$-direction (along the 2DEG boundary, see Fig. 2 for our choice of axes), $f_{n}(x)$ is the transverse amplitude profile of mode $n$, and $a_{n}$ its excitation factor. For $k_{F} L \gg 1$ the phase factors $\exp \left(i k_{n} L\right)$ vary rapidly as a function of $n$. Constructive interference of modes at the collector then requires that $k_{n} L$ differs by multiples of $2 \pi$ for a series of $n$. To find out what this condition implies for the magnetic field, we determine $k_{n}$ in WKB approximation (which should be sufficiently accurate for this purpose). 
Consider again the classical skipping orbits ( $\Gamma$ ig. 2). The position $(x, y)$ of the electron on the circle with center coordinates $\left(X, Y^{\prime}\right)$ can be expressed in terms of its velocity $\mathrm{v}$ by

$x=X+v_{y} / \omega_{c}, y=Y-v_{x} / \omega_{c}$,

with $\omega_{\mathrm{c}}=e B / m$ the cyclotron frequency. Note that the separation $X$ of the center from the boundary is constant on a skipping orbit, only the center coordinate $Y$ parallel to the houndary changes at each specular reflection. The canonical momentum of the electron is $\mathbf{p}=m \mathbf{v}-e \boldsymbol{A}$. In the Landau gauge $\boldsymbol{A}=(0, B x, 0)$ we have

$p_{x}=m v_{x}, p_{y}=-e B X$.

The wave number $k$ corresponds classically to the canonical momentum component $p_{y}=\hbar k$, sn that in view of Eq. (4) we have the correspondence $k=-(e B / h) X$. Since the motion projected on the $x$-axis is periodic, one can apply the Bohr-Sommerfeld quantization rule [21]

$\frac{1}{h} \oint p_{x} \mathrm{~d} x+\gamma=2 \pi n$.

The integral is over one period of the motion, $n$ is an integer, and $\gamma$ is the sum of the phase shifts acquired at the two turning points of the motion. The phase shift upon reflection at the boundary is $\pi$ (for an infinite barrier potential, to cnsure that incident and reflected waves cancel); The other turning point is a caustic of the skipping orbits with constant $x$, leading to a phase shift of $-\pi / 2$ [22]. This totals to $\gamma=\pi / 2$. Using also Eqs. (3) and (4) we may thus write Eq. (5) in the form

$\frac{\mathcal{c} B}{h} f(Y-y) \mathrm{d} x=2 \pi\left(n-\frac{1}{4}\right), n=1,2, \ldots N$.

This quantization rule has the simple geometrical interpretation [20] that the flux enclosed by one arc of the skipping orbit and the boundary equals $(n-1 / 4)$ times the flux quantum h/e (see insets in Fig. 6).

Eq. (6) cletermines, for a given magnetic field, the energy $E=m v^{2} / 2$ as a function of the quantum number $n$ and the wave number $k=-(c B / \hbar) X$. To carry out the integration in Eq. (6) we express $y$ in terms of $x$ by means of Eq. (3). The resulting energy spectrum $E_{n}(k)$ is given by

$\frac{2 E}{h \omega_{\mathrm{c}}}\left(\arccos \xi-\xi\left(1-\xi^{2}\right)^{1 / 2}\right)=2 \pi\left(n-\frac{1}{4}\right), \xi \equiv h k(2 m E)^{-1 / 2}$,

and is plotted in Fig. 6 (solid curves). Also plotted in Fig. 6 is the exact solution of the Schrödinger equation (dashed curves, taken from Ref. 


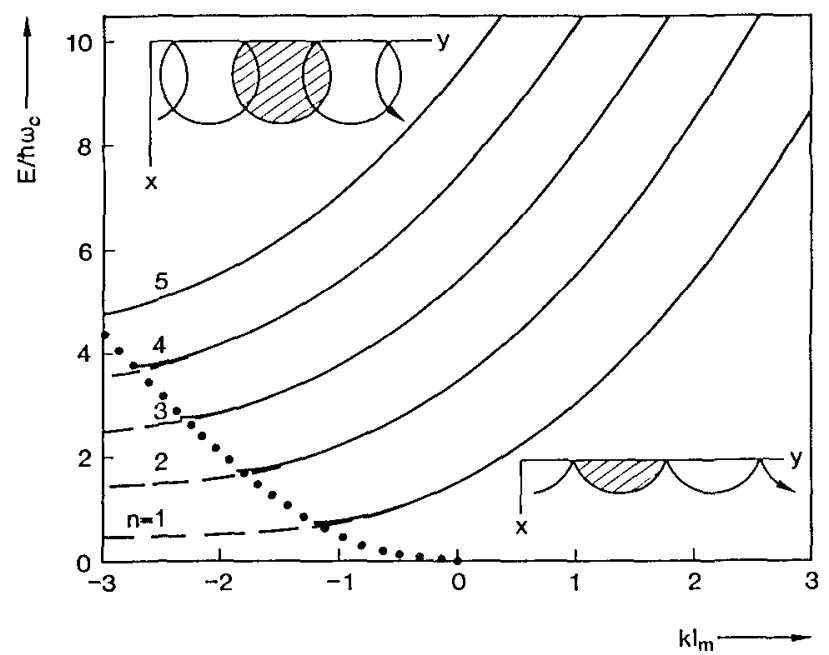

Fig.6 Energy spectı tum $\Gamma_{n}(k)$ of magnetic edge states at an infinite barier potential boundary. Note that $k I_{m}=-X / l_{m}$, with $X$ the separation of the osbital center from the boundary and $l_{m} \equiv(h / \rho B)^{1 / 2}$ the magnetic length. The insets show classical skipping orbits for positive and negative $k$. In the semi-chassical approximation the magnetic flux through the shaded areas is quantized. The sesult from Eq. (7) (solid curves) is indistinguishable from the exact solution (dashed curves, from Rer. 23), unless $k$ is within $1 / I_{m}$ of the transition from skipping to cyclotron orbits (dotted curve). [From Ref. 8.]

23). The (semi-classical) WKB approximation (7) is indistinguishable on this scale from the exact solution, except just before the transition from skipping orbits to bulk cyclotron orbits at $X=m \mathrm{~m} / \mathrm{c} B$ (dolled curve in Fig. 6). The quantized wave numbers $k_{n}$ at the Fermi energy satisfy $E_{n}\left(k_{n}\right)=E_{1}$, so that $k_{n}$ is determined by $\Gamma q$. (7) with the substitutions $E \equiv E_{\mathrm{V}}, \xi \equiv k_{n} / k_{\mathrm{V}}$. As shown in Fig. 7 the resulting dependence on $n$ of the phase $k_{n} L$ is close to linear in a broad interval,

$k_{n} L=\mathrm{constant}-2 \pi n B / B_{\text {Cocus }}+k_{\text {I }} L \times \operatorname{order}(1-2 n / N)^{3}$.

It follows from this cxpansion that if $B / B_{\text {focus }}$ is an integer, a fraction of order $\left(1 / k_{1:} L\right)^{1 / 3}$ of the $N$ edge channels interfere constructively at the collector. Because of the $1 / 3$ power, this is a substantial fraction even for the large $k_{\mathrm{r}} L \sim 10^{2}$ of the experiment. The relevant states have quantum number $n$ in an interval centered around $N / 2$, corresponding classically to skipping orbits which reach the boundary at approximately right angles. The edge states outside the clomain of linear $n$-dependence of the phase give rise to fine structure without a simple periodicity. 


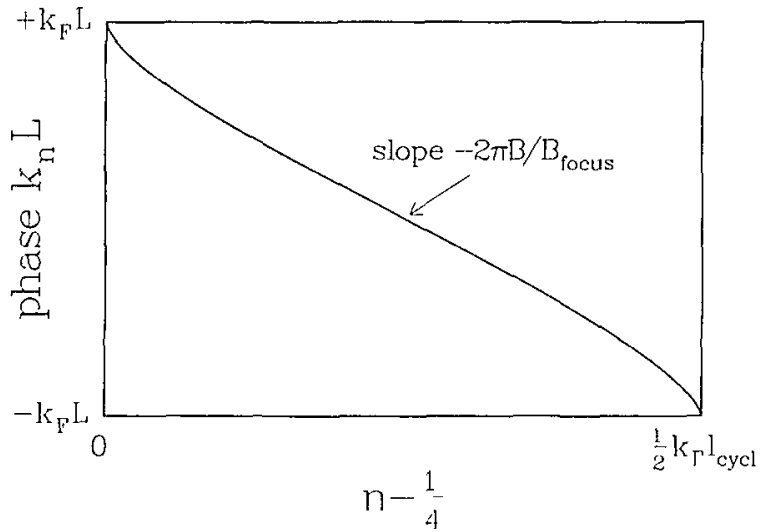

Fig. 7

Phase $k_{n} L$ of the edge channels at the collector, calculated from Eq. (7). Note the domain of approximately lincar $n-$-dependence of the phase, icsponsible for the oscitlations with $B_{\text {locus }}-$ periodicily. [From Ref'8.]

To determine the amplitude of the oscillations in the collector vollage, we need to know the excitation factors of the modes by the injector and the transmission amplitude through the collector. In Ref. 8 we calculated these quantities using a point-clipole injector and a transmission amplitude proportional to the derivative $\partial \Psi / \partial x$ of the unperturbed wave function at the collector - thereby neglecting the finite width of the injector and collector point contacts. The result obtained there can be written in the form

$$
\frac{V_{\mathrm{c}}}{l_{\mathrm{i}}}=\frac{h}{2 e^{2}}\left|\frac{1}{N} \sum_{n=1}^{N} \mathrm{e}^{\mathrm{i} k_{n} L}\right|^{2} .
$$

In Fig. 8 we have plotted the rocusing spectrum from Eq. (9), corresponding to the experimental Fig. 5. The inset shows the rourier transform for $B \geq 0.8 \mathrm{~T}$. There is no detailed one-to-one correspondence between the experimental and theoretical spectra. No such correspondence was to be expected in view of the sensitivity of the experimental spectrum to small variations in gate voltage (which defines the point contacts and the 2DEG boundary). Those features of the experimental spectrum which are insensitive to the precise measurement conditions are, however, well reproduced by the calculation: We recognize in Fig. 8 the low-field focusing peaks and the large-amplitude high-field oscillations with the same periodicity. I The reason that the periodicity $B_{\text {rocus }}$ in Fig. 8 is somewhat larger than in Fig. 5 is most likely the experimental uncertainty in the effective point contact separation of the order of the split-gate opening $(250 \mathrm{~nm})$. I The high-field oscillations range from about 0 to $10 \mathrm{k} \Omega$ in both theory and experiment. 


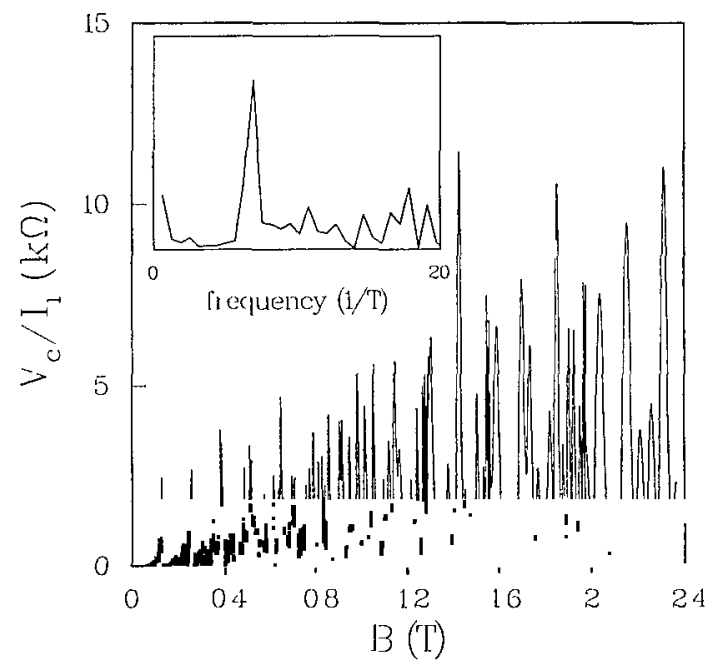

Fig.8 Focusing spectum calculated from Eq. (9), for patameters cortesponding to the experimental Fig. 5. The inset shows the Fourier tuansform for $B \geq 0.8 \mathrm{~T}$. Infinitesimally small point contact widths are assumed in the calculation.

This maximum amplitude is not far below the theoretical upper bound of $h / 2 e^{2} \approx 13 \mathrm{k} \Omega$, which follows from Eq. (9) if we assume that all the modes interfere constructively. This indicates that a maximal phase coherence is realized in the experiment, and implies that:

- The experimental injector and collector point contacts resemble the idealized point source/detcctor in the calculation;

- Scattering events other than specular scattering on the boundary can be largely ignored (since any other inclastic as well as elastic scattering events would scramble the phases and reduce the oscillations with $B_{\text {focus }}$ - periodicily).

It follows from Eq. (9) that if interference of the modes is ignored, the normal quantum IIall resistance $h / 2 N e^{2}$ is obtained. This is $n o t$ a general result, but depends specifically on the properties of the injector and collector point contacts - as we will discuss in the following section.

\section{Quantum point contacts as Landau level selectors}

Mode interference becomes unimportant if the magnetic field is sufficiently strong, and the point contacts are sufficiently wide, that the electrostatic potential in the point contact region does not cause scattering between the modes. The requirement for such adiabatic transport is that the potential varies slowly on the scale of $l_{\text {cycl }}$ (in the quantum Ilall effect regime where $N \sim 1$ and $E_{1} \sim h \omega_{c}$, the cyclotron radius is the magnetic length $\left.l_{\mathrm{m}} \equiv(h / e B)^{1 / 2}\right)$. In this fickl regime the form of the 


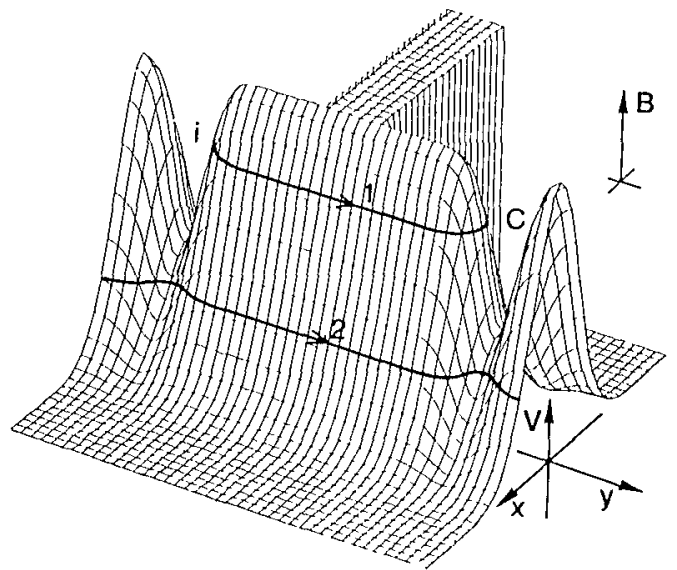

Fig. 9

Schematic potential lanclscape, showing the 2DEG boundary and the saddle-slaped injector and collector point contacts. In a strong magnetic lickl the edge channels are cxlended along equipotentials (Eq. (I0)), as indicated here for $n=1,2$ (the arrows point in the disection of motion). In this calse a Ifall conductance of $\left(2 e^{2} / h\right) N$ wilh $N=1$ would be measured by the noint contacts - in spite of the presence of 2 occupied Landau levels in the bulk 2DEG.

electrostatic potential $V(x, y)$ defining the point contacts becomes important, and the point injector/detector model used in the previous section - while adequate at lower magnetic fields - is insulficient.

Schematically, $V(x, y)$ is represented in Fig. 9. Fringing fields from the split-gate create a potential barrier in the point contacts, so that $V$ has a saddle form as shown. The heights of the barriers $E_{\mathrm{i}}, E_{\mathrm{c}}$ in the injector and collector are separately adjustable by means of the voltages on the split-gates, and can be determined from the conductances of the individual point contacts [24]. The width of the point contacts does not play a role, because it is larger than $l_{\text {cycl }}$. The adiabatic transport is along equipotentials as indicated in Fig. 9 (arrows point in the direction of motion, determined by the potential gradient). The energy of the equipotential is the gutiding center energy $E_{\mathrm{G}_{\mathrm{i}}}$, which is given for edge channel $n$ by

$E_{\mathrm{G}}=E_{\mathrm{I}}-\left(n-\frac{1}{2}\right) h \omega_{\mathrm{C}}$

(Zeeman spin-splitting of the energy levels should be included at large magnetic fields, but is ignored here for simplicity). The edge channels can only be transmitted through a point contact if $E_{\mathrm{G}}$ exceeds the potential barrier height (disregarding tunneling through the barrier). The injector thus injects $N_{1} \approx\left(E_{\mathrm{F}^{-}}-E_{\mathrm{i}}\right) / \hbar \omega_{\mathrm{c}}$ edge channels into the $2 \mathrm{DEG}$, while the collector is capable of detecting $N_{\mathrm{c}} \approx\left(E_{\mathrm{F}}-E_{\mathrm{c}}\right) / / \omega_{\mathrm{c}}$ channels. Along the boundary of the 2DEG, however, a larger number of $N \approx E_{\Gamma} / h \omega_{c}$ edge channels, equal to the number of bulk Landau levels in the 2DEG, are available for the current transport. The selective population, and detection, of Landau levels leads to deviations from the normal IIall resistance.

These considerations can be put on a theoretical basis by applying the general Landauer-Büttiker formalism $[15,16]$, which relates resistances 
to transmission probabilities into current and voltage probes. Consider the geometry in $\Gamma$ ig. 1 of a three-terminal conductor with point contacts in two of the probes. The probes are connected by perfect leads to reservoirs which have a constant electro-chemical potential. We denote by $\mu_{\mathrm{i}}$ and $\mu_{\mathrm{c}}$ the chemical potentials of the two reservoirs connected, respectively, to the injector and collector point contact, and by $\mu_{\mathrm{c}}$ the chemical potential of the third reservoir (the current drain). Following Büttiker [16], we can relate the currents $I_{\alpha}(\alpha=\mathrm{i}, \mathrm{c}, \mathrm{d})$ in the three leads to these chemical potentials via the transmission probabilities $T_{\alpha \rightarrow \beta}$ (from reservoir $\alpha$ to reservoir $\beta$ ) and reflection probabilities $R_{\alpha}$ (from reservoir $\alpha$ back to the same reservoir),

$\frac{h}{2 e} I_{\alpha}=\left(N_{\alpha}-R_{\alpha}\right) \mu_{\alpha}-\sum_{\beta \neq \alpha} T_{\beta \rightarrow \alpha} \mu_{\beta}$,

$N_{\alpha}$ being the number of occupied modes in the lead $\alpha$. We now impose the condition that the collector draws no net current, which implies $I_{\mathrm{c}}=0$ and $I_{\mathrm{d}}=-I_{\mathrm{i}}$, and choose our zero of energy such that $\mu_{\mathrm{d}}=0$. One then finds from Eq. (11) the two equations

$\mu_{\mathrm{c}}=\frac{T_{\mathrm{i} \rightarrow \mathrm{c}}}{N_{\mathrm{c}}-R_{\mathrm{c}}} \mu_{\mathrm{i}}, \frac{h}{2 e} I_{\mathrm{i}}=\left(N_{\mathrm{i}}-R_{\mathrm{i}}\right) \mu_{\mathrm{i}}-T_{\mathrm{c} \rightarrow \mathrm{i}} \mu_{\mathrm{c}}$,

and obtains for the ratio of collector voltage $V_{\mathrm{c}}=\mu_{\mathrm{c}} / e$ (measured relative to the voltage of the current drain) to injected current $I_{\mathrm{i}}$ the result

$\frac{V_{\mathrm{c}}}{l_{\mathrm{i}}}=\frac{2 c^{2}}{h} \frac{T_{\mathrm{i} \rightarrow \mathrm{c}}}{G_{\mathrm{i}} G_{\mathrm{c}}-\delta}$.

Here $\delta \equiv\left(2 e^{2} / h\right)^{2} T_{\mathrm{i} \rightarrow \mathrm{c}} T_{\mathrm{c} \rightarrow \mathrm{i}}$, and $G_{\mathrm{i}} \equiv\left(2 e^{2} / h\right)\left(N_{\mathrm{i}}-R_{\mathrm{i}}\right)$ and $G_{\mathrm{c}} \equiv$ $\left(2 e^{2} / h\right)\left(N_{\mathrm{c}}-R_{\mathrm{c}}\right)$ denote the conductances of the injector and collector point contact, respectively. The injector-collector reciprocity in electron focusing, demonstrated in Fig. 3 , is manifest in Eq. (13), since $G_{\mathrm{i}}$ and $G_{\mathrm{c}}$ are even in $B$ and $[16] T_{\mathrm{i} \rightarrow \mathrm{c}}(B)=T_{\mathrm{c} \rightarrow \mathrm{i}}(-B)$.

In the electron focusing geometry the term $\delta$ in Eq. (13) can be neglected, since $T_{\mathrm{c} \rightarrow \mathrm{i}} \approx 0$. An additional simplification is possible in the adiabatic transport regime. We consider the case that the barrier in one of the two point contacts is sufficiently higher than in the other, to ensure that electrons which are transmitted over the highest barrier will have a negligible probability of being reflected at the lowest barrier. Then $T_{\mathrm{i} \rightarrow \mathrm{c}}$ is dominated by the transmission probability over the highest barrier, $T_{\mathrm{i} \rightarrow \mathrm{c}} \approx \min \left(N_{\mathrm{i}}-R_{\mathrm{i}}, N_{\mathrm{c}}-R_{\mathrm{c}}\right)$. Substitution into Eq. (11) gives the remarkable result that the IIall conductance $G_{1 \mathrm{I}} \equiv I_{\mathrm{i}} / V_{\mathrm{c}}$ measured in the electron focusing geometry can be expressed entirely in terms of the contact conductances $G_{\mathrm{i}}$ and $G_{\mathrm{c}}$,

$G_{\mathrm{II}} \approx \max \left(G_{\mathrm{i}}, G_{\mathrm{c}}\right)$. 


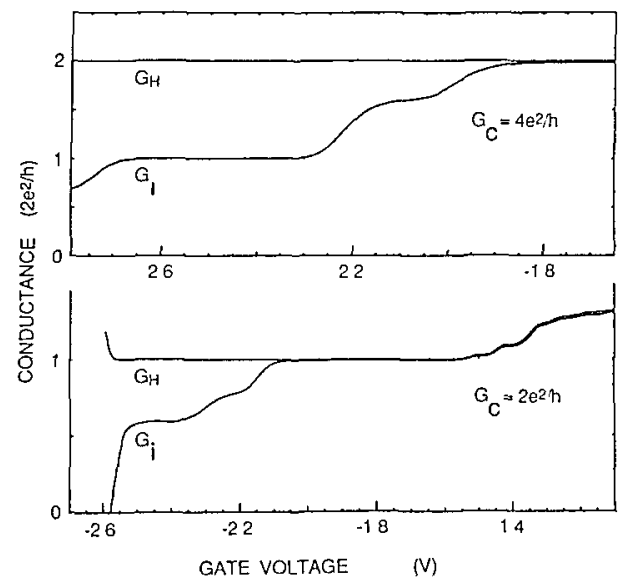

Fig. 10.

Experimental correlation betivecn the conductances $G_{1}, G_{\text {c }}$ of injector and collector, and the llall conductance $G_{11} \equiv I_{1} / V_{\mathrm{c}}$, shown to demonstiate the validity of $\mathrm{Eq} .(14)(T=1.3 \mathrm{~K}, L=$ $1.5 \mu \mathrm{m})$. The magnetic field was kept fixed (top: $B=2.5 \mathrm{~T}$, botlom: $B=3.8 \mathrm{~T}$ ). By increasing the gate voltage on one half of the split-gate defining the injector, $G_{1}$ was varied at constant $G_{\mathrm{c}}$. [ Fiom Ref 7.]

Eq. (14) tells us that quantized values of $G_{11}$ occur not at $\left(2 e^{2} / h\right) N$, as one would expect from the $N$ Landau levels in the 2DEG - but at the smaller value of $\left(2 e^{2} / h\right)$ max $\left(N_{1}, N_{c}\right)$. Moreover, there is no quantized llall conductance unless the largest of the two contact conductances is quantized. As shown in Fig. 10, this is indecd observed experimentally. Notice in particular how any deviation from quantization in $\max \left(G_{\mathrm{f}}, G_{\mathrm{c}}\right)$ is faithfully reproduced in $G_{\mathrm{II}}$. The implication of this experiment is that:

- Point contacts can be used to selectively populate and cletect Landau levels at the $2 D E G$ boundary;

- Adiabatic transport (i.e. transport in the absence of inter-Landau level scattering) has been realized over a distance of $1.5 \mu \mathrm{m}$ along the 2DEG boundary.

As discussed by Büttiker [25], the fundamental origin for deviations from the normal quantum II all effect is the absence of local equilibrium among the edge channels. Selective population is indeed an extreme example of a non-equilibrium population. Recent related experiments $[26,27]$ have demonstrated that a non-equilibrium population of edge channels can be maintained on even longer length scales, possibly as large as several hundred microns. It remains a theoretical challenge to explain these surprisingly long relaxation lengths.

\section{Conclusion}

In Sec. 1 we emphasized that the length scales relevant for the electron focusing experiment are very different in a metal and in a 2DEG. Both the ratios $\lambda_{\Gamma} / L$ and $\lambda_{\Gamma} / W$ are much larger in a $2 D E G$, typically by factors of $10^{4}$ and $10^{2}$, respectively. As we showed in Sccs. 2 and 3 , coherent electron focusing is possible in a $2 D E G$ because of this rela- 
tively large value of the Fermi wave length. The adiabatic transport discussed in Sec. 4 is also made possible by the large $\lambda_{\Gamma}$, since now $l_{\text {cycl }}=h / c B \lambda_{F}$ can become comparable to $W$ at magnetic fields of a few Tesla. To achieve the same in a metal would require fields over $100 \mathrm{~T}$. The difference in encrgy scale between a metal and a 2DEG manifests itself in the dependence of the focusing spectrum on the voltage drop over the injector. In metals, electrons are injected at energies above $E_{1:}$ which are generally much less than $E_{\mathrm{F}} \sim 5 \mathrm{eV}[28]$. In contrast, $E_{\mathrm{F}} \sim 10 \mathrm{meV}$ in a $2 \mathrm{DEG}$, and DC-biasing the small $\Lambda \mathrm{C}$ injection voltage used in the electron focusing experiment should lead to a noticeable shift in the focusing peaks, in analogy with a $\beta$-spectrometer. In the simplest model one would have (cf. Eq. (1)) $B_{\text {focus }} \propto\left(E_{\mathrm{F}}+e V_{\mathrm{DC}}\right)^{1 / 2}$, so that for a DC bias $V_{\mathrm{DC}}=1 \mathrm{mV}$ one would expect a $5 \%$ shift in the focusing periodicity - provided the hot electrons remain ballistic. This is indeed observed [29], atthough deviations from this simple behavior are found for larger DC biases (possibly related to the non-linear current-voltage characteristics of the point contacts themselves[30]). The observation of hot-electron transport over several microns is remarkable, and unexpected from related work in different systems [31].

The main result of the thenretical analysis of coherent electron focusing in Sec. 3 is the demonstration of high-field oscillations with $B_{\text {focus }}$-periodicity, but much larger amplitude than the low-field focusing peaks. This is also the feature of the experimental focusing spectra which is insensitive to small changes in gate voltage and which is found in both the devices studied. The theory can be improved in several ways. This will affect the detailed form of the spectra, but probably not the fundamental periodicity. Since the exact wave functions of the edge states are known (Weber functions), one could go beyond the WKB approximation. This will become important at large magnetic rields, when the relevant edge states have small quantum numbers. In this regime one would also have to take into account a possible $B$ - dependence of $E_{\mathrm{p}}$ : relative to the conduction band bottom (due to pinning of the Fermi energy at the Landau levels). It would be interesting to find out to what extent this bulk effect is reduced at the 2DEG boundary by the presence of edge states to fill the gap between the Landau levels.

Another direction of improvement is towards a more realistic modelling of the injector and collector point contacts. Since the maximum amplitude of the theoretical and experimental oscillations is about the same (compare Figs. 5 and 8 ), the loss of spatial coherence due to the finite point contact size does not seem to be particularly important in this experiment (infinitesimal point contact width was assumed in the calculation). On the other hand, the experimental focusing spectrum does not contain as much rapid oscillations as the calculation would predict. 
Energy averaging due to a finite temperature is not the reason for this clifference (temperatures on the order of $10 \mathrm{~K}$ are necessary to smear out the rapid oscillations). We surmise that the rapid oscillations are reduced by the collimation effect proposed originally [32] to explain the non-adclitivity of the resistance of two opposite point contacts in series [33] (and more recently invoked [34] to explain the quenching of the Hall resistance in a narrow-channel geometry [13]). Both the flaring of a point contact to form a horn, and the presence of a potential barrier in the point contact region tend to collimate the injected electron beam [32], so that electrons are predominantly injected at right angles to the boundary. The quantum mechanical correspondence discussed in Sec. 3 then implies that such a point contact excites (and detects) predominantly the edge channels with quantum number $n$ close to $N / 2$, at the expense of channels with smaller or larger $n$. Since the former edge channels are responsible for the oscillations with $B_{\text {focus }}$-periodicity, while the latter give rise to rapid aperiodic oscillations (see Fig. 7 and the accompanying discussion), the collimation effect provides one mechanism for the absence of rapid oscillations in the experimental focusing spectrum.

\section{Acknowledgement}

The authors have greatly benefitted from their collaboration with M.E.I. Brockant, C.T. Foxon, C.J.P.M. Harmans, J.J. IJarris, L.P. Kouwenhoven, P.II.M. van Loosdrecht, D. van der Marel, J.E. Mooij, M.F.II. Schuurmans, I. A. Pals, Г.M.M. Willems, and J.G. Williamson.

\section{References}

[1] Y'u.V. Sharvin, Zh.Eksp.Teor.Fiz. 48, 984 (1965) [ Sov.Phys..IETP 21, 6.55 (196.5) ]

[2] V.S. Tsoi, Pis'ma Zh.Exp.Tcor.Fiz. 19, 114 (1974) [ JETP L.clt. 19, 70 (1974)]

[3] P.C. van Son, H. van Kempen, and P. Wyder, Phys.Rev.Lelt. 58, 1567 (1987)

[4] Physics and Technology of Submicron Structures, ed. by $/$. Heinrich, $G$. Bater, and F. Kuchar (Springer, Berlin 1988); Nanostructure Physics and Fabrication, ed. by M. Reed and W.P. Kirk (Academic Press, New York, to be published)

[5] H. van Honten, B.J. van Wees, J.E. Mooij, C.IV.J. Beonakker, I.G. Williamson, and C.T. Foxon, Europhys.Lett. 5, 721 (1988)

[6] C.W.J. Beenakker, H. van Houten, and B.J. van Wees, Europhys.Lett. 7, 359 (1988)

[7] B.J. van Wees, E.M.M. Willems, C.J.P.M. IIarmans, C.W..J. Beenakker, II. van Houten, J.G. Williamson, C.T. Fo ron, and J.J. Harris, Phys.Rev.Letl. 62 $1181(1989)$ 
[8] H. van Ilouten, C.W.J. Benakker, J.G. William.son, M.E.I. Broekaart, P.H.M. wan Loosdrecht, B.J. van Wecs, I.E. Mooij, C.T. Foxon, and J.J. Harris, Phys.Rev. B (April 15, 1989)

[9] T.J. Thomton, M. Pepper, II. Almed, D. Andrews, and G.J. Davies, Phys.Rev.Lett. 56, 1198 (1986); H.Z. Zheng, H.P. Hei, D.C. Tsui, and G. Weimann, Phys. Rev. B34, 5635 (1986)

[10] B.J. wan Wees, H. wan Houten, C.W.J. Beenakker, J.G. Williamson, L.P. Konwenhoven, D. van der Marel, and C.T. Foxon, Phys.Rev.Lelt. 60, 848 (1988)

[11] D.A. Wharam, T.J. Thornton, R. Newbury, M. Pepper, H. Ahmed, J.E.F. Frost, D.G. Hasko, D.C. Peacock, D.A. Ritchie, and G.A.C. Iones, I.Phys. C21, L209 (1988)

[12] H van Houten and C.W.J. Beenakker, in: Analogies in Optics and Microelectronics, ed. by $\mathrm{I}$. van Hacringen and D. Lenstra (Kluwer, Deventer, to be published)

[13] G. Timp, A.M. Chang, P. Mankiewich, R. Behringer, J.E. Cumingham, T.Y. Chang, and R.E. Howard, Phys.Rev.Lctt. 59, 732 (1987); M.L. Ronkes, A. Scherer, S.J. Allen, Jr., H.G. Craighead, R.M. Ruthen, E.D. Beebe, and J.P. Harbison, Phys.Rev.Lct. 59, 3011 (1987); C.J.B. Ford, T.J. Thornton, R. Newbury, M. Pepper, H. Ahmed, D.C. Peacock, D.A. Ritchie, I.E.F. Frost, and G.A.C. Jones, Phys.Rev. B38, 8518 (1988); G. Timp, in: Mesoscopic Phenomena in Solids, ed. by P.A. Le'e, R.A. Webb, and B.L. Al'tshuler (Elsevier, New York, to be published)

[14] G. Timp, H.U. Baranger, P. deVegvar, J.E. Cunningham, R.E. Howard, R. Behringer, and P.M. Mankiewich, Phys.Rev.Lett. 60, 2081 (1988); $Y$. Takagaki, K. Gamo, S. Namba, S. Ishida, S. Takaoka, K. Murase, K. Ishibashi, and Y. Aoyagi, Solid State Comm. 68, 1051 (1988)

[15] R. Landamer, IBM I.Res.Dev. 1, 223 (1957); 32, 306 (1988); Z.Phys. B68, 217 (1987)

[16] M. Biittiker, Phys.Rev.Lett. 57, 1761 (1986); IBM J.Res.Dev. 32, 317 (1988); Sec also: A.D. Stone and A. Szafer, IBM J.Res.Dev. 32, 384 (1988)

[17] P.A.M. Benistant, G.F.A. van de Walle, H. van Kempen, and P. IVyder, Phys.Rev. B33, 690) (1986)

[18] M. Büttiker, Phys.Rev. B38, 12724 (1988)

[19] R.E. Prange and T.-W. Nee, Phys.Rcv. 168, 779 (1968)

[20] M.S. Khaikin, Adv.Phys. 18, 1 (1969)

[21] A.M. Kosevich and I.M. Lifshitz, Zh.Eksp.Teor.Fiz. 29, 743 (1955) [Sov.Phys.JETP 2, 646 (1956)]

[22] L.D. Landau and E.M. Lifshitz, The Classical Theory of Ficlds (Pergamon, Oxford 1987) $\S 54$

[23] A.H. Mar'Donald and P. Streda, Phys.Rev. B29, 1616 (1984)

[24] B.J. van Wees, L.P. Kouwenhoven, H. van IIouten, C.W.J. Beenakker, J.E. Mooij, C.T. Foxon, and J.J. Harris, Phys.Rev, B38, 3625 (1988)

[25] M. Büttiker, Phys.Rev. B38, 9375 (1988)

[26] S. Komiyama, H. Hirai, S. Sasa, and S. Hiyamizu (preprint)

[27] B.J. van Wees, E.M.M. Willems, L.P. Kouwenhoven, C.J.P.M. Harmans, J.G. Williamson, C.T. Foxon, and J.J Harris Phys.Rev. B (April 15, 1989)

[28] P.C. van Son, II. van Kempen, and P. Wyder, J.Phys. F17, 1471 (1987)

[29] J.G. Williamson ot al. (unpublished)

[30] L.P. Konwenhoven, B.J. yan Wees, C.J.P.M. Harmans, J.G. Williamson, H. van Houten, C.W.J. Beenakker, C.T. Fo.ron, and J.J. Harris, Phys.Rev. B (April 15, 1989) 
[31] A. Palevski, M. Heiblum, C.P. Umbach, C.M. Knoedler, A.N. Broers, and R.H. Koch (preprint)

[32] C.W.J. Beenakker and H. van Houten, Phys.Rev. B (to be published)

[33] D.A. Wharam, M. Pepper, H. Ahmed, J.E.F. Frost, D.G. Hasko, D.C. Peacock, D.A. Ritchie, and G.A.C. Jones, J.Phys. C21, L887 (1988)

[34] II.U. Baranger and A.D. Stone (preprint); A.M. Chang and T.Y. Chang (preprint); C.I.B. Ford, S. Washbum, M. Büttiker, C.M. Knoedler, and J.M. Hong (preprint) 


\section{Extract of the Foreword}

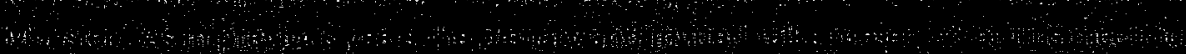

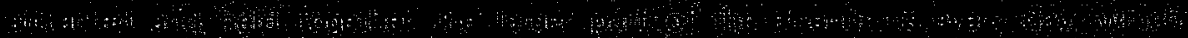

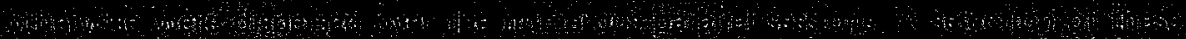

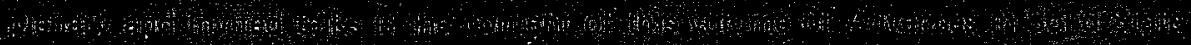

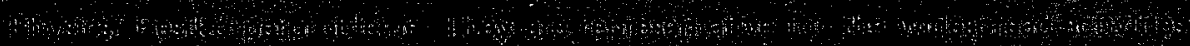

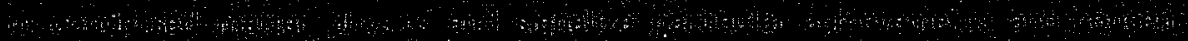

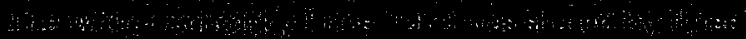

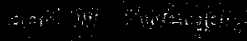

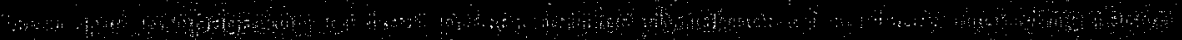

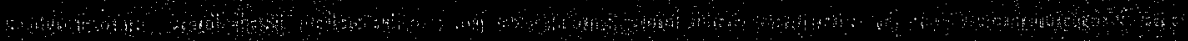

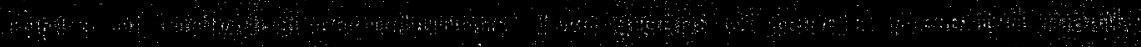




\section{FESTKÖRPERPROBLEME 29}

\section{ADVANCES IN SOLID STATE PHYSICS}

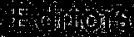

now

6.t.

का

Hit.

1)

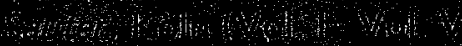

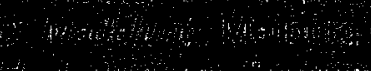

$4 \sec$

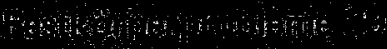

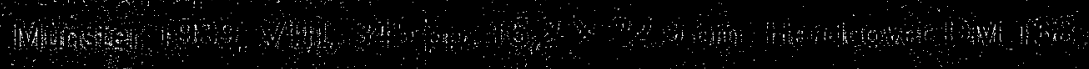

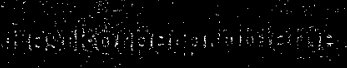

andmontos

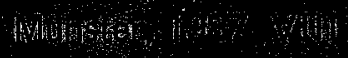




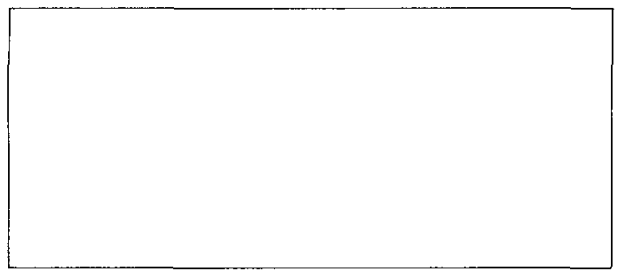

Friedr. Vieweg \& Sohn Verlagsgesellschaft $\mathrm{mbH}$ P.O.B. 5829

D-6200 Wiesbaden

Federal Republic of Germany 
Please type

or print in

capital

letters

your

name and

address

Please send immedıately

\begin{tabular}{l|l|l}
\hline No. of copies & Title & Price \\
\hline & $\begin{array}{l}\text { Festkörperprobleme 29 } \\
\text { Advances in Solid State Physics }\end{array}$ & DM 168,- \\
\hline & Festkorperprobleme 28 & DM 98,- \\
\hline & Festkorperprobleme 27 & DM 178,- \\
\hline & Festkorperprobleme 26 & DM 178,- \\
\hline & Festkorperprobleme XXII & DM 124,- \\
\hline & Festkórperprobleme XXI & DM 148,- \\
\hline & Festkorperprobleme $X X$ & DM 128,- \\
\hline
\end{tabular}

